\title{
Use of Videography for Traffic Surveys in Sri Lanka
}

Traffic Surveys are crucial for different transport studies like Origin-Destination studies, Traffic Volume estimations, Vehicle Flow characteristics Determinations, Speed and Delay Studies, Turning Movement analysis, Parking Analysis etc. Currently, Sri Lankan researchers are using mostly human-based manual surveys and semi-automated methods for Traffic Surveys. However, there is an issue in cost, effort, the value of time, and the accuracy of data gathering in the above methods. Therefore, under Accelerating Higher Education Expansion and Development (AHEAD) grant, we are researching the possibility of doing surveys using videography and Artificial Intelligence technologies for continuous and accurate data collection to reduce the burden that is currently facing.

Using CCTV video cameras, we have done surveys in different locations in Western Province to check the possibility of implementing the developed system in Sri Lanka. Fig. 1 elaborates the basic setup of the study. Four cameras were used simultaneously for 12 hours in a particular location. Fig. 2 indicates actual scenarios in the field survey.

After data collection from this CCTV video footage, videos are processed through a DeepSORT algorithm for vehicle tracking and customized trained YOLOv4 model for detecting vehicles by vehicle type.

The developed model also provided the individual vehicle speed in addition to the identification of the vehicle type [1]. These speeds are an improvement to Google speeds since it carries the vehicle type too. The classified vehicle count from the custom trained YOLOv4 model has provided better accuracy than on-site manual counting [2], [3].

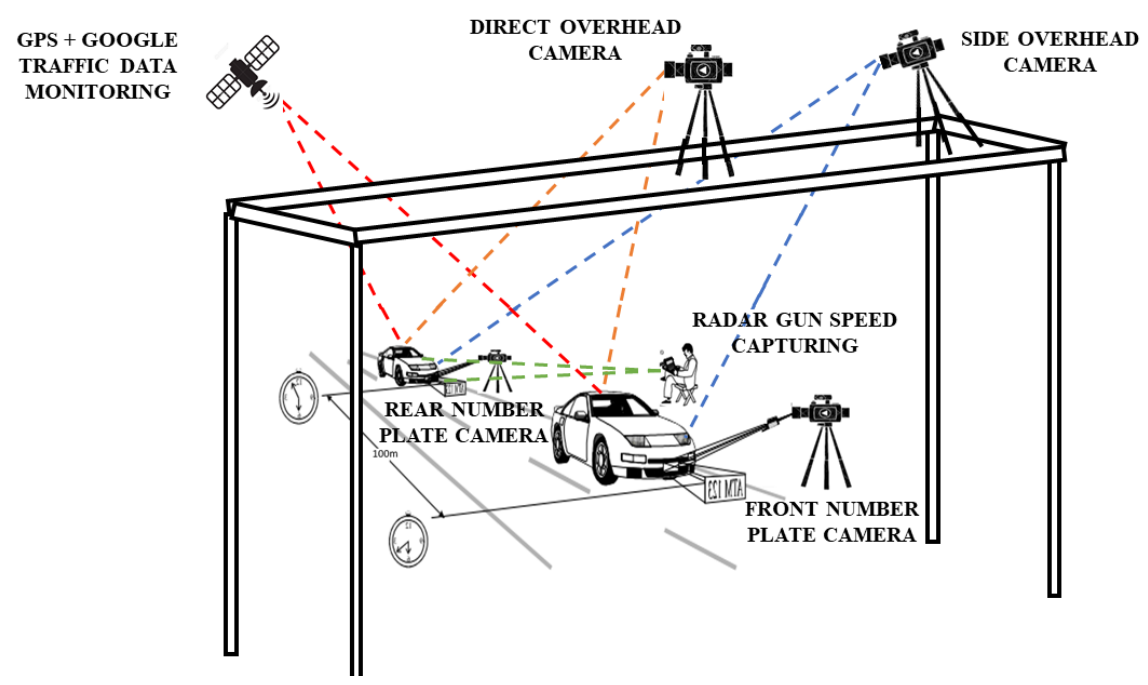


Compared with the previous studies, one of the major improvements in this study is the number of vehicle categories identified from this method (Fig. 3). We can identify seven vehicle categories which include all the types of vehicles available in the South Asian region, especially in Sri Lanka (car, three-wheeler, motor bicycle, van, bus, truck, heavy vehicles with multi-axles). Therefore, this method is a sustainable and accurate alternative to manual traffic data collection as it avoids human error and adds precision to all the traffic parameters. To improve the accuracy of this method, we require more video surveillance data with more variations in traffic conditions to feed the model to increase the input level and its variety for better prediction.
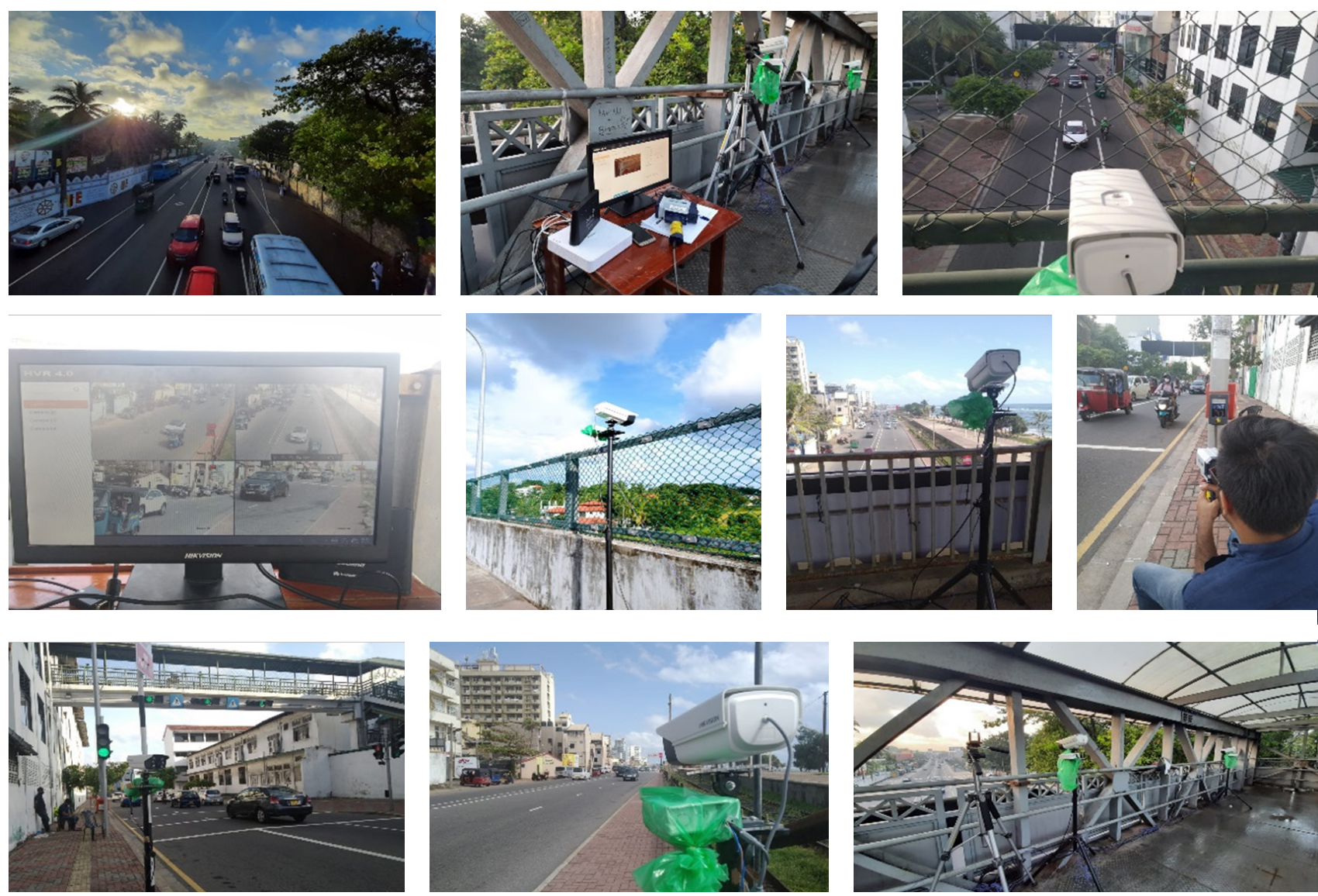

A Figure. 2. Field Survey

$\checkmark$ Figure 3. YOLOv4 Model Output

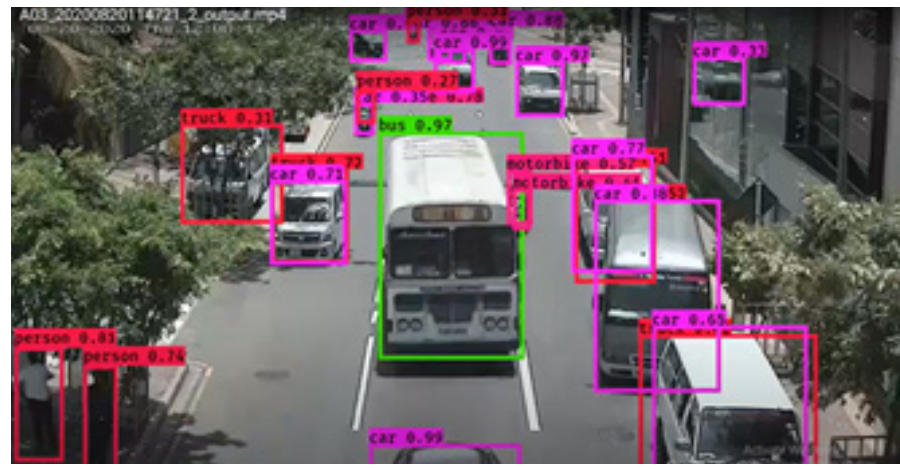

REFERENCES

[1] A. Bochkovskiy, C. Y. Wang, and H. Y. M. Liao, "YOLOv4: Optimal Speed and Accuracy of Object Detection," arXiv, 2020.

[2] B. Chethan Kumar, R. Punitha, and Mohana, "YOLOv3 and YOLOv4: Multiple object detection for surveillance applications," Proc. 3rd Int. Conf. Smart Syst. Inven. Technol. ICSSIT 2020, no. Icssit, pp. 1316-1321, 2020, doi: 10.1109/ICSSIT48917.2020.9214094.

[3] V. Mandal, A. R. Mussah, P. Jin, and Y. Adu-gyamfi, "Artificial Intelligence Enabled Traffic Monitoring System," no. September, 2020, doi: 10.20944/preprints202009.0725.v1.

Article by

Oshadhi K. Herath', Praveen Perera', T. Sivakumar', Buddhi Ayesha², Amal S. Kumarage', Amal Shehan Perera²

'Department of Transport and Logistics Management, Faculty of Engineering, University of Moratuwa, Sri Lanka.

${ }^{2}$ Department of Computer Science and Engineering, Faculty of Engineering, University of Moratuwa, Sri Lanka. 